\section{Venus could have a large magnetic moment}

\section{from Peter J. Smith}

TEN to fifteen years ago the only planets thought to possess internal magnetic fields were the Earth and Jupiter. But the data provided by space probes have transformed this picture-so much so that planetary fields must now be regarded as the rule rather than the exception. The strongest known field is still that of Jupiter which has a magnetic moment of $1.4 \times 10^{30}$ gauss $\mathrm{cm}^{3}$. Then comes the Earth with a moment of $8 \times 10^{25}$ gauss $\mathrm{cm}^{3}$, Mercury with $5 \times 10^{22}$ gauss $\mathrm{cm}^{3}$ and Mars with $2.5 \times 10^{22}$ gauss $\mathrm{cm}^{3}$. The Moon (admittedly not a planet), on the other hand, has no detectable internal dipole, which means to say that if it has an internal field at all the moment must be lower than $10^{19}$ gauss $\mathrm{cm}^{3}$.

The absence of a lunar field is explicable on the basis of the Moon's small core and its low rate of rotation. More surprising, however, especially in view of the fields discovered on the other terrestrial planets, is the apparent lack of magnetism on Venus. According to Dolginov et al. (Kosmich. Issled, 7,$747 ; 1969$ ) the upper limit of a barely detectable Venusian moment must be $5 \times 10^{21}-8 \times 10^{21}$ gauss $\mathrm{cm}^{3}$. Yet on the grounds of size alone Venus would be expected to have a core about as large as that of the Earth (and thus larger than that of Mercury), and its rate of rotation is only about four times lower than that of Mercury. On this basis Venus ought to have a moment of $1.9 \times 10^{23}$ gauss $\mathrm{cm}^{3}$, or so a forthcoming report predicts. Why, then, has a magnetic field greater than the Dolginov limit not been observed?

The first attempt to measure the global field of Venus was by Mariner 2 in 1962. No field was detected at the closest approach of 6.6 radii-a result which placed an upper limit of $4 \times 10^{24}$ gauss $\mathrm{cm}^{3}$ on the Venusian moment. Five years later the flyby of the USA's Mariner 5 apparently enabled this limit to be reduced to $8 \times 10^{22}$ gauss $\mathrm{cm}^{3}$, a figure derived from the observed position of the bow shock. But in the same year (1967) Russia's Venera 4, which landed on the planet's surface, measured the magnetic field directly, down to an altitude of $200 \mathrm{~km}$. It was these data, combined with those from Mariner 5, which Dolginov and his colleagues used to reduce the maximum possible moment to $5 \times 10^{21}$ $8 \times 10^{21}$ gauss $\mathrm{cm}^{3}$, the lowest upper limit yet proposed.

But Russell (Geophys. Res. Lett.,
3,125 ; 1976) now claims that an alternative interpretation of the Venera 4 data is possible. Limiting his analysis to the last $10 \mathrm{~min}$ of the field measurements (representing altitudes of less than $5,000 \mathrm{~km}$ ), Russell shows that for three different models of the solar wind-Venus interaction there is a strong dependence of magnetic field on altitude, with the field increasing in strength towards the planetary surface. Moreover, for two of these models the altitude dependence is close to an inverse cube law, the exception to this being the least likely model which assumes no well-developed magnetosphere. In both cases the extrapolated surface field is about $30 \mathrm{nT}$, which would be equivalent to a magnetic moment of $6.5 \times 10^{22}$ gauss $\mathrm{cm}^{3}$.

In short, the altitude dependence is consistent with the existence of a planetary field, and the magnitude of the field suggests that the internal moment, if there is one, is larger than that of either Mars or Mercury. The qualification is needed here, however, because the form of the Venera 4 data, though necessary, is not sufficient to prove the presence of an internal global field. There are alternative interpretations. The magnetic observations could, for example, be explained not only in terms of an internal field but also of a field induced in the ionosphere. The rejection of one or other of the options will probably have to await further data, preferably from a Venus orbiter. But in the meantime a Venusian moment of at least $10^{22}$ gauss $\mathrm{cm}^{3}$ must be regarded as at least plausible and, if Venus is not to be exceptional, even likely.

\section{Genu valgum, copper and fluorosis in India}

\section{from Zaka Imam}

First reported in 1973 (Krishnamachari and Kamala Krishnaswamy, Lancet, ii, 877-897), a syndrome characterised by genu valgum (knock knees) and osteoporosis of long bones is widely prevalent in certain parts of Andhra, and in February 1976 some cases were also being reported from Tamil Nadu and Karnataka states in India. There are three important aspects to the occurrence of this syndrome-it is restricted to regions endemic for fluorosis, the victims are mainly under $25 \mathrm{yr}$ old and there is no mention of this syndrome in the reports by earlier workers who studied fluorosis in those regions of Andhra. The syndrome therefore seems to be a manifestation of fluoride toxicity that has emerged only during the past two decades. But the syndrome does not occur in all fluorotic regions of the states in question, nor in fluorotic regions in many other parts of the Indian subcontinent.

Although the cause of this geographical variation in the manifestation of fluoride toxicity is not yet fully understood, accumulated evidence provides some explanation. The regions where genu valgum is prevalent are mostly fluorotic regions in the vicinity of dams constructed during the past two decades. In Andhra state the syndrome is mainly confined to villages within a radius of $80 \mathrm{~km}$ from the Nagarjunasagar dam where there is more than 3.00 p.p.m. fluoride in the drinking water. Villages more than $50 \mathrm{~km}$ from the Hopset dam (Karnataka state), mostly with more than 1.00 p.p.m. fluoride in the water, have no cases of genu valgum, but villages within a radius of $5-30 \mathrm{~km}$ of this dam, about $50 \%$ of which have more than 1.00 p.p.m. fluoride in the water, have a few affected children. The syndrome is also prevalent in villages within $20 \mathrm{~km}$ of the Aliyar dam in Tamil Nadu, where the fluoride content in the water is fairly high, the minimum level being 1.9 p.p.m. The prevalence of the syndrome seems to decrease with increasing distance from the dam.

In 1974 the National Institute of Nutrition in Hyderabad suggested that the construction of large water reservoirs near areas endemic for fluorosis may be related to the development of this syndrome. The mechanism involved is not clear. It is known that the construction of large water reservoirs considerably influences the ecology of the surrounding areas by changing the soil conditions for several kilometres from the site of construction. The increased subsoil water subsequently changes the trace element composition of food grains grown on such soil and brings about changes in the trace element composition of water. The level of subsoil water in parts of Andhra where genu valgum is widely prevalent has risen in recent years. An increase in the subsoil water also increases soil alkalinity, producing conditions in which plants are known to pick up more molybdenum from the soil. In this context it is interesting that molybdenum is found in the higher range of concentrations in sorghum and rice grown in fluorotic regions. Sorghum is the staple of the poorer sections of the community and generally contains twice as much molybdenum as does 